\title{
Exploring How to Conduct Infection Prevention and Control Education in Undergraduate Nursing Programs in Korea: Focus Group Interview Analysis
}

\author{
Chang, Sung $\mathrm{Ok}^{1)} \cdot$ Sohng, Kyeong-Yae ${ }^{2)} \cdot \mathrm{Kim} \mathrm{Kyunghe}^{3)} \cdot$ Won, Jongsoon $^{4)}$. \\ Choi, Min-Jung ${ }^{5)} \cdot$ Chaung, Seung-Kyo ${ }^{6}$
}

\author{
1) Professor, College of Nursing, Korea University, Seoul \\ 2) Professor, College of Nursing, The Catholic University of Korea, Seoul \\ 3) Professor, College of Nursing, Red Cross College of Nursing, Chung-Ang University, Seoul \\ 4) Professor, College of Nursing, Eulji University, Seongnam \\ 5) Research Instructor, College of Nursing, The Catholic University of Korea, Seoul \\ 6) Professor, Department of Nursing, Semyung University, Jecheon, Korea
}

\begin{abstract}
Purpose: In the nursing profession, it is imperative that students are able to transfer their undergraduate knowledge and skills into practice to become competent nurses. The aim of this study was to illuminate how infection prevention and control (IPC) education would be conducted in undergraduate nursing programs. Methods: A qualitative design utilizing focus group interviews as its data collection method was employed. Twelve professors from twelve South Korean universities that have undergraduate nursing programs were recruited as research participants and divided into two focus groups. Results: Focus group interview analysis showed that IPC education in undergraduate nursing programs for fostering IPC competency was composed of two categories: a pre-clinical course and a clinical course. Each included three subcategories-education contents, education strategies, and considerations of how infection control is taught to students as they continue from beginning to advanced-and the themes of each subcategory were derived. Conclusions: The findings of this study can provide an overview of how nursing professors should teach IPC education to undergraduates. As IPC education for nurses is very important, more in-depth discussions that include educators, clinical mentors, and nursing students regarding IPC education are needed to ensure patients' safety in clinical settings.
\end{abstract}

Key Words: Infection; Prevention and control; Education; Students; Nursing

주요어: 감염, 예방과 관리, 교육, 간호대학생

1) 고려대학교 간호대학 교수

2) 가톨릭대학교 간호대학 교수

3) 중앙대학교 적십자간호대학 교수

4) 을지대학교 간호대학 교수

5) 가톨릭대학교 간호대학 연구강사

6) 세명대학교 간호학과 교수

Received Apr 20, 2019 Revised Aug 7, 2019 Accepted Aug 12, 2019

Corresponding author: Chaung, Seung-Kyo Department of Nursing, Semyung University

65 Semyung-ro, Jecheon 27136, Korea

Tel: +82-43-649-1351, Fax: +82-43-649-7051, E-mail: chaungck@hanmail.net 


\section{INTRODUCTION}

Korea has been implementing an accreditation system for medical institutions since 2010[1]. At the same time, the number of Korean medical institutions receiving Joint Commission International (JCI) accreditation has been increasing. Significantly for this study, one of the assessments of patient safety in medical institution accreditation is aimed at "reducing the risk of infection"[2]. Infection prevention and control (IPC) is a necessary element of daily practice in many health professions to safeguard patients and staffs [3]. In addition, hospitals are using infection experts to establish a patient safety culture, and infection control is an important factor in hospital accreditation $[4,5]$. Therefore, nursing educators are well aware that IPC education is very important in undergraduate education [6].

Previous research on IPC education has largely divided into studies focused on IPC education strategies utilizing standardized patient, simulation environments, or actual clinical situations for IPC education [7-9] and studies focused on the IPC-education elements such as microbiology, hand washing, healthcare-associated infection with standard precautions, preventive measures for medical personnel, and issues on patient immunity such as HIV/ AIDS patients [10-16].

However, one report argued that there was a debate about what education would be desirable to teach infection control in the undergraduate course of health sciences [17]. This report focuses on the complexity of interconnected elements for professional training in undergraduate health sciences and suggested that IPC teaching should be based on an educational strategy that stimulates students' reflection and encourages them to develop critical thinking about their experiences.

However, the findings from studies suggest that the undergraduate nursing curriculum lacks adequate IPC training $[18,19]$. Studies emphasizing the weaknesses of health professionals' knowledge and behavior in relation to IPC demonstrate that one of the implications for this problem is in lack of theory and practice in the initial training of these professionals. The current IPC education in an undergraduate nursing program does not specify how the IPC theory is applied and linked to clinical practice through each clinical course.

Therefore, it emphasizes the need to discuss IPC teaching techniques in the undergraduate course in health science [20]. It is necessary to explore how nursing educators conduct IPC education to nurture future nurses and equip them with competencies directly applicable to medical institutions. This study aimed to identify and explore how IPC education would be conducted in undergraduate nursing programs.

Therefore, this study is expected to contribute to the awareness of nursing instructors about how infectious education is emphasized and how it is linked and how infection education should be done in each clinical subject through undergraduate nursing curriculum.

\section{METHODS}

\section{Research Design}

A qualitative design utilizing focus group interviews as its data collection method was employed to explore nursing educators' in-depth thoughts and opinions on effective IPC education in undergraduate nursing programs. Focus group interviews are more effective than individual interview collections. The synergies and interaction elements between group members are useful for drawing in-depth opinions that can be mutually agreed on a particular topic and are a useful methodology for deriving desirable ideas for a particular topic [21].

\section{Research Participants}

A purposive sample was utilized to explore the thoughts and opinions of the nursing professors who volunteered for this study. The researchers sought to derive an overview of IPC education by recruiting professors in charge of supervising different nursing subjects engaged in different undergraduate nursing programs. Twelve professors from twelve South Korean universities that have undergraduate nursing programs were recruited as research participants and divided into two focus groups. Focus Group 1 was consisted of six professors: one specializing in child nursing, one in maternal nursing, two in adult nursing, one in the fundamentals of nursing, and one in nursing administration. Focus Group 2 was consisted of six professors: one specializing in child nursing, one in adult nursing, one in the fundamentals of nursing, two in community health nursing, and one in gerontological nursing. In order to share and discuss the characteristics of IPC education in each nursing major, professors from each major in nursing were assigned to each focus group.

The study participants were interested in nursing education to enhance prevention and control of infection, and they expressed their opinions regarding improvements in IPC education based on their educational experiences. 
Table 1. Characteristics of Participants

\begin{tabular}{|c|c|c|c|c|}
\hline \multirow{2}{*}{ ID } & \multicolumn{2}{|c|}{ Focus group 1} & \multicolumn{2}{|c|}{ Focus group 2} \\
\hline & Teaching career (year) & Major & Teaching career (year) & Major \\
\hline 1 & 10 & Maternity nursing & 27 & Adult health nursing \\
\hline 2 & 22 & Adult health nursing & 12 & Gerontological nursing \\
\hline 3 & 19 & Fundamental nursing & 13 & Community health nursing \\
\hline 4 & 4 & Pediatric nursing & 8 & Pediatric nursing \\
\hline 5 & 20 & Fundamental nursing & 19 & Community health nursing \\
\hline 6 & 16 & Adult health nursing & 8 & Fundamental nursing \\
\hline
\end{tabular}

They understood the purpose of our research and agreed to participate in one of the two focus groups. The characteristics of Group 1 were an average age of 49.0 years old and average educational experience of 15.2 years, while the characteristics of Group 2 were an average age of 50.7 and average educational experience of 14.5 years (Table 1 ).

\section{Data Collection}

Data collection was conducted from August to October 2017 in a conference room of a university. Focus group interviews were carried out once for 120 minutes. Researchers sent out the questions by e-mail to the participants a week before the interview in order to facilitate a more substantive discussion. In order to elicit valuable opinions from the participants, researchers reviewed the researches on IPC and nursing education and organized their questions for the focus group interviews into three categories: 1) IPC education strategies, 2) IPC educational elements, and 3) other educational considerations on IPC education.

The focus group interviews were conducted based on the questions that were sent in advance and began with an introduction on the necessity of consensus among nursing educators for delineating proper education modalities to promote students' IPC competencies. The focus group interview was carried out by a researcher who had extensive experience in qualitative research and in focus group interview. The focus group interview process was attended by all the researchers of this study to facilitate interaction so participants could explore diverse opinions. While debriefing, researchers confirmed with participants that the group discussions were properly conducted for each question, confirmed whether there were any objections to the opinions raised, and agreed that the discussion on the topic was complete before the next question was asked.

\section{Ethical Considerations}

The study was approved by the Institutional Review Board of the university (IRB No. 1040548-**-IRB-17-65-A-1), and permission to conduct the research was obtained from each participant. The purpose of the study was explained to all subjects, who were informed that their participation was voluntary.

\section{Data Analysis}

Many of the research methodologies that use focus group interviews adopt a sort of qualitative content analysis and consider the group as an analytical unit [22]. Conventional qualitative content analysis was used to gain an understanding of the perspectives of nursing educators on how IPC education would be conducted in undergraduate nursing programs. Data analysis was conducted according to conventional qualitative content analysis [23]. In the analysis of the data, the researchers read the raw data of the interview until they became familiar. And then the meaningful data were reviewed until the initial codes were generated. Then the researchers identified categories and subcategories. Condensed meaning units were tentatively grouped into subthemes of each subcategory through several discussions. The latent subthemes were formulated into themes. Finally, the researchers identified categories, subcategories and created the themes.

\section{Trustworthiness}

In this study, to ensure the reliability of the study, the researchers summarized the discussions generated by each focus group interview and received the confirmation from all participants by summarizing the agreed upon opinions. To ensure the rigor of qualitative research, the 
strategies of Padgett [24] was used. We organized interview guidelines for consensus building and confirmed the research purpose with all the professors participating in the focus group interviews. Then, we collected interview data with audio recording. We have done peer-debriefing during data analysis to avoid biased analysis and constantly compared the results by member check

\section{RESULTS}

The focus group interview analysis showed that IPC education in undergraduate nursing programs for fostering infection control competency was composed of two categories with six subcategories, from which the themes of each subcategory were derived. Participants in this study presumed that IPC education in an undergraduate nursing program was divided into two categories, pre-clinical and clinical courses. Under each category were three subcategories: education contents, education strategies, and how IPC would be taught to students as they progressed from freshmen just beginning their studies to seniors preparing to graduate (Figure 1).

\section{Infection Prevention and Control Education in Pre-clinical Courses}

In this study, pre-clinical courses indicate nursing edu- cation before clinical placements. The fundamentals of nursing, in which nursing education starts, was mainly mentioned among participants and consisted of three categories: education contents, education strategies and consideration of linkage between two levels of education: microbiology and fundamentals of nursing. Education contents were presented as a lecture course and laboratory practice.

\section{1) Education contents: Lecture courses}

(1) Conceptualization of the infection cycle based on its transmission path

It was emphasized among participants that the setting of patient safety when approached by a medical professional is the main focus of the lecture on infectious diseases, which starts from a conceptual view and moves to IPC strategies within the fundamentals of nursing. Participants suggested that those strategies are based on the theory of the cycle of infection and transmission and how to take precautions against it when caring for patients.

Infection prevention and control strategies within the fundamentals of nursing are based on the theory of the cycle of infection and transmission of diseases. When you think about the chain of infection, the first basic principle in caring for patients is to break each link in the chain of infection... (FG (Focus Group) 2, 6 (ID))

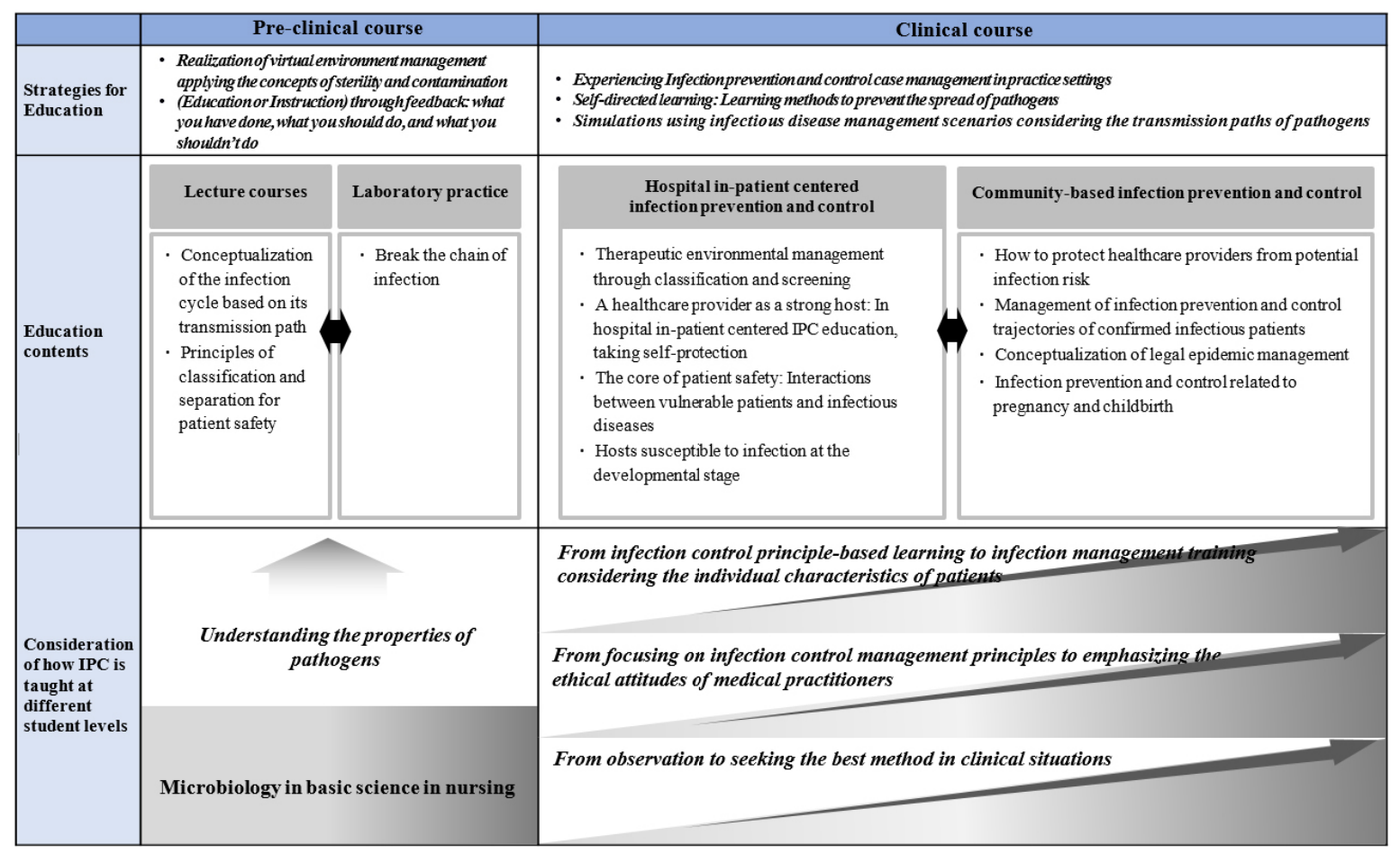

Figure 1. An exploration on how to conduct infection control education in undergraduate nursing program. 
(2) Principles of classification and separation for patient safety

Participants emphasized that nurses must recognize that standardized precaution measures for IPC, which are basically compliance requirements of clinicians, they also suggested nurses ultimately divide, classify, and manage people and objects to promote patient safety in clinical settings. Therefore, participants emphasized that it is important to learn that the principles of classifying and managing are based on the virulence and potency of microorganisms, the susceptibility of the host, the ability of microorganisms to live in the host and the potential for crossinfection among medical personnel, which are the principles of effective IPC.

Infection prevention and control education in the fundamentals of nursing usually starts from the most basic step, hand hygiene, and education is one of the ways to establish the standard precautions and transmission-based precaution concepts most commonly applied in clinical situations (FG 1, 3).

\section{2) Education contents: Laboratory practice}

(1) Break the chain of infection

Participants emphasized that the focus of IPC education for laboratory practice would be the student's conceptualization of isolation and protective isolation. Therefore, they suggested that students could learn by repeating the actions of medical practitioners, such as hand hygiene and gowning, that break the chain of infection.

The most important aspect of infection control is to break the chain of infection. Therefore, we will focus on the principles of basic nursing care that meet the requirements for patients' safety: hand hygiene, isolation, disinfection, and sterilization (FG 2,6).

\section{3) Strategies for Education}

(1) Realization of virtual environment management

It was suggested among participants that the strategy to implement nursing management in a virtual environment in the laboratory setting is to apply the concepts of aseptic conditions and contamination state.

The strategy of infection prevention and control education in the fundamentals of nursing is to learn to develop a therapeutic environment by understanding and applying techniques to maintain medical and surgical asepsis, remembering to take patients' needs into account (FG 2, 6).
(2) What you have done, what you should do, and what you shouldn't do

It was agreed among participants that the main role of the instructor in IPC education is to educate students about the guidelines for standard and transmission-based precautions when caring for patients in the virtual environment in laboratory, to demonstrate related nursing actions in front of students, and to provide students with feedback on what and what not to do in a virtual clinic.

Students will be taught how to manage infection in diverse circumstances through individual feedback in the context of isolation from infection and situations corresponding to protective isolation (FG 1, 5).

\section{4) Consideration of linkage between two levels of education} in pre-clinical: microbiology and fundamentals of nursing Those themes were identified as considerations of linkage between microbiology and fundamentals of nursing through focus group discussions

(1) Understanding the properties of pathogens

The property of linkage between the microbiology in basic nursing science and fundamentals of nursing of education was raised among participants in terms of teaching how to break the chain of infection in the fundamentals of nursing, an introductory nursing class, as well as an understanding of the properties of pathogens in basic microbiology before students take more advanced courses.

The goal of learning microbiology is to understand the causative organisms behind patients' infectious diseases first and second to prevent the spread of such causative organisms to other patients by knowing the transmission pathway characteristics. Knowledge of the properties of microbiological pathogens should be linked to infection control education in the fundamentals of nursing (FG 1, 5).

\section{Clinical Courses}

In this study, the clinical course indicates nursing education with clinical placements for practical study. Participants agreed that the IPC education in the clinical courses is focused on hospitals and communities.

\section{1) Education Contents: Hospital in-patient centered infec-} tion prevention and control

The IPC education in the hospital environment is focused on hospitalized patients. The following themes were 
mentioned and agreed upon in the focus groups.

(1) Therapeutic environmental management through classification and screening

The main focus of IPC was on standard precautions which are the main focus of hospital IPC. The subject of clinical infection management was emphasized among participants; future nursing students should be aware of the invisible pathways in which bacteria grow and propagate in the treatment process.

Particularly, we should emphasize the knowledge of contact care related to multidrug-resistant bacteria (MRSA and VRE) and the role of infectious disease management in the clinical field (FG 1, 1).

In addition, the purpose of IPC was emphasized in education regarding infectious diseases in order to control the chances of patients becoming infected and to maximize the effects of treatment. Participants suggested that the educational focus was on establishing the concept of isolating contaminated areas and aseptic areas and putting it into practice.

How to manage patients' fluids in clinical practice, how to identify areas within the ward according to their degree of contamination, and related topics should also be taught in clinical practice (FG 1, 1).

(2) A healthcare provider as a strong host

In hospital in-patient centered IPC education, taking self-protection measures as a medical professional is emphasized among participants. This is because the hospital environment can be viewed as a collection of diverse bacteria, and thus measures to protect healthcare personnel from potential risks caused by these bacteria should be included in IPC education.

Needlestick injuries are a common clinical event. All health care providers must be vaccinated to prevent infection by pathogens, and nursing students should be immunized before they can practice (FG 1, 6).

(3) The core of patient safety: Interactions between vulnerable patients and infectious diseases

Since hospitals are places where vulnerable patients are hospitalized for treatment and where various pathogens are concentrated at the same time, participants emphasized that interactions between vulnerable patients and infectious diseases should be integrated into all nursing care for infection prevention and control.

When dealing with diseases of immunocompromised patients, such as aplastic anemia or leukemia, the focus should be primarily on infection control methods (FG1, 2).

(4) Hosts susceptible to infection at the developmental stage

Participants emphasized that how to create a safe environment for children and the elderly who are especially vulnerable to infection should be included in clinical practice education. Clinical training should also include how nurses manage patients who are susceptible to infection with impaired immune systems due to disease.

In the case of children and in consideration of the developmental stage they inhabit, immunization vaccinations are a very important element of infection prevention and control education. Nursing students need to know in advance what should be done to prevent rotaviruses before they begin neonatal practice, along with other areas they should be careful about. Moreover, in pediatric nursing infection prevention and control in children with leukemia is very important (FG 1, 4).

It is important to check carefully whether an elderly patient is infected because the symptoms of such infections are different from those of adult patients. In addition, nutritional management is very important for infection prevention and control, especially in nursing homes (FG 2, 2).

\section{2) Education Contents: Community-based infection pre-} vention and control

Since in community-based clinical practice, IPC education is mainly carried out in homes, schools, industrial facilities, and public health settings. The following topics were discussed among the participants and suggested as constituents of IPC education.

(1) How to protect healthcare providers from potential infection risk

It was emphasized among participants that measures to protect healthcare providers from potential infectious diseases should be included in community IPC education as well as in hospital-centered IPC education.

When visiting a home, a student may get a needlestick injury when doing a diabetes check. Students al- 
so need to know proper needle care after giving such tests. Nursing students can meet a carrier at any time in their community. It should also be emphasized that nursing students should strengthen their personal immunity (FG 2, 5).

In addition, participants suggested that the process of identifying and managing infectious patients should be included in IPC education for community nursing education, unlike hospital-based nursing education.

Patients with tuberculosis, hepatitis C, and HIV/ AIDS patients are carriers in their communities. Students must be taught how to protect themselves when they are practicing in community settings. Gonorrhea and other conditions are also related to infectious transmission (FG 2, 3).

(2) Management of infection prevention and control trajectories of confirmed infectious patients

It has been suggested among participants that a management trajectory incorporating IPC principles of infectious patients already identified and managed by community health facilities should be included in IPC education.

If a patient with active tuberculosis visits a public health center and not well-controlled, the nurses in the health center's tuberculosis room will have the nursing students put on masks. Students are experiencing a situation in which the patient is infected and can spread the disease (FG 2, 5).

(3) Conceptualization of legal epidemic management

It was suggested among participants that knowledge of the legally regulated management principles of infectious diseases that can spread in a community and the way to prevent them from spreading should be studied in the undergraduate curriculum.

A professor teaches how infectious disease management is regulated legally and teaches infection control in connection with quality of water or air pollution. In particular, nursing students should learn how to prevent communicable diseases and prevent the spread of infectious diseases, especially in schools and industrial settings (FG 2,3).

The management of infectious diseases due to the movement of people from country to country has been empha- sized among participants as learning contents to keep up with globalization.

When a disease spreads across Korea, such as MERS, which originated in the Middle East, we have to deal with issues such as how to cope with infectious diseases rapidly spreading to widespread locations (FG $2,3)$.

(4) Infection prevention and control related to pregnancy and childbirth

Participants emphasized that one of the management of vulnerable nursing clients in the community was on maternal IPC related to pregnancy and childbirth. In particular, it was suggested that the management of infectious diseases affecting the fetus and post-partum management should be covered in IPC education.

Students must learn how to manage perinatal infections and to manage infections such as the chick virus, which affects the fetus and is emerging as a global problem. This is a problem that needs to be addressed in maternal nursing (FG 2, 4).

\section{3) Strategies for Education}

In clinical courses, the following themes regarding IPC education strategies were suggested.

(1) Experiencing infection prevention and control case management in practice settings

The most emphasized educational strategy among participants was the necessity of organizing nurses' education so that students can directly experience case management reflecting the clinical elements of infection management in the field.

In clinical practice training, it is effective for infection prevention and control education to connect students with hospital infection prevention and control centers to, for example, let students know why hand hygiene is important in clinics and whether bacteria really survive after hand hygiene (FG 1, 1).

(2) Self-directed learning: Learning methods to prevent the spread of pathogens

The instructor should enable students to form a concept of IPC. As a way to prevent the propagation of pathogens, it was suggested among participants that IPC measures be clearly defined so that self-directed education is accompanied by an effective learning strategy. 
How to prevent infection from spreading, such as hand hygiene, or teaching what is UTI management? Let's make a simple movie about IPC situation. I think this kind of learning has been very helpful to students (FG 1, 2).

(3) Simulations using infectious disease management scenarios considering the transmission paths of pathogens

Participants suggested that simulation training can help ensure that infection management is carried out in parallel with caring for patients by integrating IPC education into clinical case management scenarios and having students study them.

Since respiratory infections are the most prevalent in childhood patients, patient nursing scenarios are created for respiratory infections or digestive tract infections. In this way, we can educate our students to ensure that infection control is always integrated into patient care (FG 1, 2).

\section{4) Considerations of linkage among the level of clinical courses in IPC education}

In general, undergraduate nurses earn a four-year degree. However, among participants, there is no consensus on how to determine and link the levels of IPC education, so it is necessary to determine the level of IPC education among different year students. Participants discussed how to achieve this, and the themes of their considerations on the topic were identified.

(1) From infection control principle-based learning to infection management training considering the individual characteristics of patients

Participants suggested that the progress of education in the clinical courses has been suggested to be one of transition from the on-site confirmation of the general principles of infection prevention and control, such as the cycle of infection and precaution standards, to dealing with specific conditions associated with infected patients.

In the first year, students learn about infectious microorganisms and inflammation in microbiology. In their second year, they learn basic infection control principles in the fundamentals of nursing. They then move on to major nursing subjects such as adult, maternity and community health nursing, and take practicums in hospital and community facilities where they can experience infection control management in various disease situations. In their senior year, it is necessary to create scenarios centered on MERS or tuberculosis patients for teaching how to protect and manage individual patients in various situations from infection prevention and control perspectives (FG1,5).

(2) From focusing on infection prevention and control management principles to emphasizing the ethical attitudes of medical practitioners

Participants emphasized that in applying IPC principles in clinical nursing care, a transition from low level simply focusing IPC management principles to higher level dealing with ethical aspect regarding IPC in education should be done in order to learn healthcare providers' ethical attitudes in connection with IPC.

It is important to practice nursing according to infection prevention and control principles. However, it is very important to educate the ethical aspects related to infection prevention and control, and how a nurse should report to hospitals and patients when he or she has made an error related to infection (FG1,1).

(3) From observation to seeking the best method in clinical situations

Participants suggested that IPC education would be taught in such a way that students learn to seek the best IPC method, e.g., setting priorities in terms of infectious disease management, starting from observing real-life infection management in clinical practice and moving to seek best nursing practices by applying IPC principles.

If students observe the infection management cases of various patients in clinical practice, they will have an integrated view of infection prevention and control when seeking the best way in clinical practice (FG $1,1)$.

\section{DISCUSSION}

We attempted to explore an overview for infection control education in undergraduate nursing programs centered on nursing educators because the actions developed by professors are one of the main sources of influence in IPC training process among the educational modalities that students glimpse during their training.

A previous study suggests that to boost reflection and critical thinking of the student, IPC education should be emphasized before entering the fields of practical activities [17]. The findings of this study showed that nursing 
educators classified IPC education as pre-clinical and clinical which is before and after nursing students' clinical placement. They emphasized that IPC education in preclinical should be educated by microbiology and fundamental of nursing with emphasizing that microbiology should be taught prior to the fundamentals of nursing. This study suggested a systematic ordering of infection education which can be meaningful suggestions in the undergraduate nursing curriculum. In addition, the IPC education strategies presented in this study suggested the educational approach of IPC education from the lower grades to the upper grades in the undergraduate nursing curriculum. In the nursing education, the IPC education should be done through the whole undergraduate course, and these findings could be a reference factor to consider.

We tried to organize a focus group panels to include professors teaching major areas of nursing. Since a professor who is well versed in individual nursing subjects may not be aware that $\mathrm{s} / \mathrm{he}$ is responsible for IPC education as well. A previous study that investigated how the topic of IPC was addressed over the four years of an undergraduate nursing course only found that, in the second, third, and fourth years, there was mention regarding IPC in the documents [25]. In addition, a study suggested that it was difficult to identify the differences in infectious control education at the undergraduate level and at the clinical nurse level in the studies [26]. Thus, it is difficult for clinician mentors to guide students in various clinical settings to guess what level of IPC education students are receiving [11]. Studies emphasized the need to teach IPC education to be an exclusively transversal subject in the curriculum of course in all its phases aiming to understand the amplitude and complexity of the subject by the student $[17,26]$. In order to explore IPC education in the undergraduate nursing curriculum, we tried to include focus group panels in different universities and different nursing major areas, and this attempt was the first attempt in Korean nursing education.

In addition, we sought to suggest potential strategies to improve the IPC competency of undergraduate nursing students in this study. When competent graduates are deployed, we expect that their work will contribute to desirable outcomes and that they will implement life-saving practices. Previously, various studies suggested educational strategies for the effectiveness of IPC education, as well as presenting the contents of infection control education. However, those studies have not provided nursing educators with an overall view of how IPC education in undergraduate programs would be conducted. The IPC practices of students were positively influenced primarily by their university education and the good practices of their clinical mentors [27]. However, studies indicate there may be deficits in the education provided to nursing students in relation to infection prevention and control [15, 28]. In addition, one study suggests deficits related to IPC education are perceived differently by students and mentors [29].

Nurses bear a major responsibility for IPC as part of their daily patient care activities. They play a major role in ensuring that appropriate practices are in place to meet the infection control standards in their institutions [30]. Therefore, competency in IPC is a crucial component for implementing best practices for nurses to ensure patient safety and provide high-quality care [10,11]. In addition, nurses represent the largest group employed in health care, and they have the greatest degree of contact with and risk of infection from patients [12]. During clinical training, nursing students are at even more risk of infection because they are inexperienced [13]. Therefore, effective IPC practices should not only be a topic for nurses' in-service continuing education but also part of the essential knowledge and skills imparted in undergraduate nursing education [30].

In this study, we attempted to present an overview (Figure 1) of how IPC education should be done in the undergraduate nursing curriculum which was not examined in past research. As IPC education in the nursing undergraduate curriculum is very important, more in-depth discussions that include educators, clinical mentors, and nursing students are needed from a more integrative viewpoint to ensure patients' safety in clinical settings.

This study is an exploratory study to be elaborated through many studies in the future. However, the findings of this study might promote the debate about the teaching IPC education in undergraduate courses in nursing, and contributes to professionals of major areas in nursing to analyze the current reality and glimpses new ways to strengthen IPC education in the nursing curriculum.

The data in this study were obtained from a convenient sample of professors who work in twelve universities that have undergraduate nursing programs rather than from a randomly selected sample from undergraduate nursing programs in Korea. However, we targeted universities with nursing education programs that have nursing graduates working in hospitals that are nationally and internationally accredited. Participants may not have been representative of other professors in other undergraduate nursing programs. Additionally, the participants of this study may have had a particular interest in providing their opinions about IPC education in undergraduate nursing programs. 


\section{CONCLUSION}

This study provides an overview of how IPC education would be conducted in undergraduate nursing programs. Today, due to the rapidly escalating antibiotic resistance and the growing number of immunocompromised patients at risk of acquiring infections, knowledge in an appropriate application of IPC practice is becoming significantly important for all healthcare workers. The findings of this study lay a basis for future discussions on developing a standardized framework for IPC education in undergraduate nursing education.

\section{REFERENCES}

1. The Korea Institute for Healthcare Accreditation. About KOIHA: History [Internet]. Seoul: The Korea Institute for Healthcare Accreditation; 2010 [cited 2018 May 11]. Available from: http://www.koiha.or.kr/member/en/contents/ensub01/en sub01_04.do

2. Joint Commission International. Hospital Search [Internet]. Illinois: World Hospital Search; 2017 [cited 2018 April 10]. Available from:

https://www.worldhospitalsearch.org/hospital-search/?F_ Search=South\%20Korea

3. Cox JL, Simpson MD, Letts W, Cavanagh H. Re-thinking microbiology/infection control education to enhance the practice-readiness of health professional students: more than just a curriculum issue. Journal of Learning Design. 2015;8:55-67. https://doi.org/10.5204/jld.v8i1.224

4. Jalali A, Dinmohammadi MR, Ali-Mohammadpor S, Mohammadi M, Mahbobi M. National accreditation and experiences of hospitals' infection control specialists-a qualitative study. Journal of Nosocomial Infection. 2014;1(1):7-17.

5. Sakamoto F, Sakihama T, Saint S, Greene MT, Ratz D, Tokuda $Y$. Health care-associated infection prevention in Japan: the role of safety culture. American Journal of Infection Control. 2014;42(8):888-893. https://doi.org/10.1016/j.ajic.2014.05.018

6. Chiasson M. Exploring infection prevention and control education in Atlantic Canadian undergraduate nursing education programs [master's thesis]. Labrador: Memorial University of Newfoundland; 2015. p. 132-139.

7. Devenny A, Lord D, Matthews J, Tuhacek J, Vitlip J, Zhang M, et al. Avstick: An intravenous catheter insertion simulator for use with standardized patients. Nursing Education Perspectives. 2018;39(2):102-104

8. Castro-Sanchez E, Drumright LN, Gharbi M, Farrell S, Holmes $\mathrm{AH}$. Mapping antimicrobial stewardship in undergraduate medical, dental, pharmacy, nursing and veterinary education in the United Kingdom. PLoS One. 2016;11(2):e0150056. https://doi.org/10.1371/journal.pone.0150056

9. Diaz-Pena R, Irissont-Duran JA, Barrera de Leon JC. Level of knowledge of and capability for application of isolation precautions in a tertiary-level pediatric hospital. Gaceta Médica de Mexico. 2015;151(5):567-575.

10. Yano R, Okubo T, Shimoda T, Matsuo J, Yamaguchi H. A simple and short microbiology practical improves undergraduate nursing students' awareness of bacterial traits and ability to avoid spreading infections. BMC Medical Education. 2019;19 (1):53. https:// doi.org/10.1186/s12909-019-1483-4

11. Liu LM, Curtis J, Crookes PA. Identifying essential infection control competencies for newly graduated nurses: a three-phase study in Australia and Taiwan. The Journal of Hospital Infection. 2014;86(2):100-109.

https://doi.org/10.1016/j.jhin.2013.08.009

12. Cox JL, Simpson MD, Letts W, Cavanagh HM. Putting it into practice: infection control professionals' perspectives on early career nursing graduates' microbiology and infection control knowledge and practice. Contemporary Nurse. 2014;49(1):8392. https://doi.org/10.1080/10376178.2014.11081957

13. Fica CA, Jemenao PM, Ruiz RG, Larrondo LM, Hurtado HC, Munoz GG, et al. Biological risk accidents among undergraduate healthcare students: five years experience. Revista Chilena de Infectologia. 2010;27(1):34-39.

https://doi.org/10.4067/S0716-10182010000100005

14. Al-Sheyab NA, Obaidat MM, Bani Salman AE, Lafi SQ. Toxoplasmosis-related knowledge and preventive practices among undergraduate female students in Jordan. Journal of Food Protection. 2015;78(6):1161-1166. https://doi.org/10.4315/0362-028x.Jfp-14-579

15. Aniaku JK, Amedonu EK, Fusheini A. Assessment of knowledge, attitude and vaccination status of hepatitis $\mathrm{B}$ among nursing training students in Ho, Ghana. Annals of Global Health. 2019; 85(1). https://doi.org/10.5334/aogh.750

16. Ozakgul AA, Sendir M, Atav AS, Kiziltan B. Attitudes towards HIV/AIDS patients and empathic tendencies: a study of Turkish undergraduate nursing students. Nurse Education Today. 2014;34(6):929-933. https://doi.org/10.1016/j.nedt.2013.10.018

17. Massaroli A, Jussara Gue M, José Luis Medina M, Julia Valéria de Oliveira Vargas B, Kenya Schmidt R, Mariely Carmelina B. Teaching of infection control in undergraduate courses in health sciences: opinion of experts. Revista Brasileira de Enfermagem. 2018;71:1626-1634.

https://doi.org/10.1590/0034-7167-2017-0928

18. Hassan ZM. Improving knowledge and compliance with infection control standard precautions among undergraduate nursing students in Jordan. American Journal of Infection Control. 2018;46(3):297-302. https://doi.org/10.1016/j.ajic.2017.09.010 
19. Ojo OO, Ojo O. Assessing students' knowledge of healthcareassociated infections: a global perspective. British Journal of Nursing. 2017;26(20):1121-1126.

https://doi.org/10.12968/bjon.2017.26.20.1121

20. Ward DJ. The barriers and motivators to learning infection control in clinical placements: interviews with midwifery students. Nurse Education Today. 2013;33(5):486-491. https://doi.org/10.1016/j.nedt.2012.05.024

21. Dilshad RM, Latif MI. Focus group interview as a tool for qualitative research: an analysis. Pakistan Journal of Social Sciences. 2013;33(1):191-198

22. Carey MA. Comment: concerns in the analysis of focus group data. Qualitative Health Research. 1995;5(4):487-495. https://doi.org/10.1177/104973239500500409

23. Hsieh H, Shannon SE. Three approaches to qualitative content analysis. Qualitative Health Research. 2005;15(9):1277-1288. https://doi.org/10.1177/1049732305276687

24. Padgett DK. Does the glove really fit? Qualitative research and clinical social work practice. Social Work. 1998;43(4):373-381. https://doi.org/sw/43.4.373

25. de Oliveira Giroti SK, Garanhani ML. Infections related to health care in nurses' education. Revista da Rede de Enfermagem do
Nordeste. 2015;16(1):64-71.

https://doi.org/10.15253/2175-6783.2015000100009

26. Ward DJ. The infection control education needs of nursing students: an interview study with students and mentors. Nurse Education Today. 2011;31(8):819-824. https://doi.org/10.1016/j.nedt.2010.12.017

27. Zimmerman PA, Gilbert J, Brown L, Shaban RZ. Infection prevention and control learning and practice in pre-registration undergraduate nursing: the sociological influences of the clinical environment. Infection, Disease \& Health. 2016;21(3):131-132. https://doi.org/10.1016/j.idh.2016.09.069

28. Labrague LJ, McEnroe-Petitte DM, van de Mortel T, Nasirudeen AMA. A systematic review on hand hygiene knowledge and compliance in student nurses. International Nursing Review. 2018;65(3):336-348. https://doi.org/10.1111/inr.12410

29. Ward DJ. The role of education in the prevention and control of infection: a review of the literature. Nurse Education Today. 2011;31(1):9-17. https://doi.org/10.1016/j.nedt.2010.03.007

30. Cummins D, Muldoon J. Informing and educating undergraduates on HIV. Australian Nursing and Midwifery Journal. 2014;21(9):51. 